\title{
La materia Cine y publicidad en los estudios sobre Publicidad y Relaciones Públicas. Propuesta de planificación y metodología docente
}

\author{
Silvia GARCíA MIRÓN \\ Universidad de Vigo \\ silviamiron@uvigo.es
}

\begin{abstract}
Resumen
La disciplina publicitaria se caracteriza, entre otras cuestiones, por su interacción con numerosas áreas de conocimiento. Entre ellas, consideramos clave la materia Cine y publicidad en el proceso curricular del alumno con la finalidad de comprender las distintas interrelaciones entre estos dos ámbitos. Tras observar su presencia en los planes de estudio de las distintas titulaciones de Publicidad y RRPP en España, incluimos una posible guía docente que cubre un amplio abanico de competencias con un planteamiento metodológico a partir de un aprendizaje a través de tareas o proyectos.
\end{abstract}

Palabras clave: Cine; Publicidad; Guía docente; Planificación docente; ABP (Aprendizaje Basado en Problemas).

\section{The subject of Cinema and advertising in Advertising and Public Relations studies. A proposal of teaching planning and methodology}

\begin{abstract}
The advertising discipline is characterized, among other issues, by its interaction with many áreas of knowledge. Among them, we consider as a key matter the subject Cinema and advertising in the student's curriculum process in otrder to understand the different relationships between these two áreas. After observing its presence in the curricula of the different degrees of Advertising and Public Relations in Spain, we include a posible teaching guide that covers a wide range of skills from a methodological approach based on the PBL (Problem-based learning) technique
\end{abstract}

Key words: Cinema; Advertising; teaching guide; teaching planning; PBL (Problem-based learning).

\section{Referencia normalizada:}

García Mirón, S. (2013) La materia Cine y Publicidad en los estudios sobre Publicidad y Relaciones Públicas. Propuesta de planificación y metodología docente. Historia y Comunicación Social. Vol. 18 No Especial Octubre. Págs. 119-131.

Sumario: 1.Introducción. 2. Metodología. 3. Situación actual de la materia sobre Cine y publicidad en la titulación de Publicidad y RRPP. 4. Metodología docente, planning y sistema de evaluación en la oferta de la Uvigo. 4.1. Descripción de la metodología docente utilizada. 4.2. Sistema de evaluación del alumno y planificación temporal. 5. Conclusiones. 


\section{Introducción}

La adaptación al Espacio Europeo de Educación Superior (EEES) al que han tenido que enfrentarse el cómputo de universidades españolas supuso un conjunto de ajustes en diversos ámbitos, entre ellos en la propia estructura de las titulaciones así como en la inclusión de fórmulas innovadoras por parte de los docentes en el proceso de enseñanza. En este contexto la formación académica se reorienta a través de la incorporación en la planificación docente de una mayor implicación por parte del alumno en su proceso de aprendizaje.

Estos cambios también han venido de la mano de la revisión de la oferta de títulos en la Universidad española y de los planes de estudio. En este sentido, en el caso de Publicidad y Relaciones Públicas cada centro (ya sea de carácter público o privado) ha tratado de incluir aquellas necesidades que esta disciplina requiere, como la incorporación de conocimientos de distintas áreas necesarias para que el futuro egresado en publicidad gestione de la forma más eficaz posible aquellos planteamientos que se le requieran en términos de comunicación publicitaria. Nos referimos a áreas como la psicología, sociología, economía, marketing, estadística, etc.; pero también a cuestiones relativas a la cultura y tradiciones específicas de cada país o el desarrollo de los medios. Las materias transversales son una constante en la oferta de grados en comunicación. Entendemos que los contenidos sobre Cine y Publicidad (a los que nos referiremos más adelante) resultan claves dentro del proceso curricular del alumno al producirse no sólo un conocimiento de este medio sino también de las posibilidades que surgen a través del cine como medio publicitario o el uso que desde la actividad cinematográfica se realiza de la publicidad.

Así, teniendo en consideración estas ideas previas, afrontaremos con este artículo varios objetivos. En primer lugar, nos detendremos en conocer la situación actual en la que se encuentra la materia Cine y publicidad en la oferta generada por las universidades españolas. A partir de ahí, y de ser el caso, llevaremos a cabo una comparativa entre los distintos epígrafes que componen las guías docentes de la materia en cuestión en cada uno de los centros en los que se oferte. Como segundo gran objetivo nos planteamos ofrecer una solución de adaptación de esta materia al EEES a través de una planificación estructurada a partir del ABP (Aprendizaje Basado en Problemas). Es decir, se trabaja y se lleva a cabo una evaluación del alumno a través del planteamiento de distintos proyectos a lo largo del semestre así como otros estudios de caso más concretos durante las sesiones prácticas, dando respuesta a algunas de las principales competencias que el alumno necesita incorporar a su currículo académico. Para dar respuesta a este objetivo detallaremos la finalidad de la materia, los planteamientos metodológicos docentes (junto con la descripción y cronograma de cada una de las tareas), el sistema de evaluación empleado y las posibilidades de atención personalizada de las que dispone el alumno 


\section{Metodología}

En relación con la metodología aplicada debemos hacer mención, previamente, a dos factores que influyen en la elaboración de esta investigación y que han sido destacados por Torres Romay (2006: 613-620): por una parte la saturación de oferta formativa en el campo de la comunicación y, por otra parte, la complejidad y desigualdad que se observa en la aplicación del proceso de adaptación a estos estudios, concretamente en relación con los centros públicos y privados, siendo estos últimos los que trazan diferentes opciones en su oferta - creando títulos propios o incluso dobles o triples titulaciones - con la finalidad de resultar más competitivos y atractivos ante el alumnado. Por otra parte, cada universidad ha podido definir políticas curriculares propias, que pueden servir como elemento definitorio de cada centro, lo que conforma su competitividad en el mercado de titulaciones universitarias y su principal herramienta para medir la calidad de su oferta.

En cuanto al proceso hemos optado por la elaboración de una muestra a la que se aplica un análisis de tipo descriptivo con finalidad exploratoria siguiendo una estructura de problema-solución. Así, tras comprobar la situación de los planes de estudio de las carreras de comunicación y las guías docentes (de haberlas) de las materias que podemos agrupar bajo la denominación de Cine y Publicidad, proporcionaremos una posible solución a la organización y contenidos de la materia siguiendo el modelo de enseñanza basado en el ABP.

De acuerdo con los objetivos planteados consideramos como hipótesis principal la idea de que la tendencia profesionalizante de los nuevos estudios universitarios ha supuesto que se tenga en cuenta en los planes de estudio los contenidos sobre Cine y Publicidad en la oferta de materias optativas del grado en Publicidad y RR.PP. A ello añadimos que las cualidades del aprendizaje a través de proyectos favorecerán una enseñanza de tipo dinámica para el alumno

\section{Situación actual de la materia cine y publicidad en la titulación de publicidad y rrpp}

La comunicación vive una situación de protagonismo en el contexto actual, lo que se representa en la oferta de titulaciones universitarias con un amplio abanico si bien congregadas a través de tres titulaciones clave: Periodismo, Publicidad y RR.PP. y Comunicación Audiovisual ${ }^{1}$. No obstante, en los centros privados pueden encontrarse títulos propios alejados, en cierto modo, de estas tres disciplinas tradicionales. Estas titulaciones siguen encontrándose entre las más demandadas y, por ende, mantienen algunas de las notas de corte más elevadas. De ello podemos extraer el hecho de que la comunicación es un ámbito de gran interés para los estudiantes que acceden a estudios superiores universitarios. 
Tras la investigación realizada hemos encontrado varios datos que debemos dar a conocer. Para comenzar, es preciso destacar que de las 74 universidades que conforman el conjunto español son 54 (32 públicas y 22 privadas) las que ofrecen titulaciones relacionadas con la comunicación (cfr. Tabla 1). De ese número, Publicidad y RRPP se imparte en 28 universidades, de las cuales 15 se corresponden con centros públicos

Tabla 1. Información sobre el modelo universitario español

\begin{tabular}{|l|c|}
\hline Tipo & $\mathbf{N}^{\mathbf{0}}$ \\
\hline Universo total (total universidades en España) & 74 \\
\hline Universidades públicas & 49 \\
\hline Universidades privadas & 25 \\
\hline Total universidades con titulaciones de comunicación & 54 \\
\hline Total universidades que imparten Publicidad y RRPP & 29 \\
\hline Universidades públicas que imparten Publicidad y RRPP & 15 \\
\hline $\begin{array}{l}\text { Titulaciones de Publicidad y RR.PP. que incluyen contenidos de Cine y } \\
\text { publicidad }\end{array}$ & 1 \\
\hline
\end{tabular}

Fuente: Elaboración propia

De entre las cifras reseñadas, nos centraremos en algunos datos con mayor deta1le. Para comenzar, recogemos en las siguientes tablas la relación de todas aquellas universidades de carácter público ${ }^{2}$, y privado ${ }^{3}$ que ofrecen estudios de comunicación. Han sido resaltadas aquellas universidades que incluyen estudios específicos en Publicidad, que son las que conforman nuestra muestra.

En relación con la información referida a las universidades públicas (cfr. Tabla 2) advertimos varias cuestiones que deben ser reseñadas como cambios en las denominaciones clásicas u ofertas de dobles grados. En primer lugar, en la Universidad de Girona, la denominación del grado en Comunicación Audiovisual se establece como Audiovisual y Multimedia y en la Politécnica de Catalunya hace lo propio con Medios Audiovisuales; mientras que Miguel Hernández D’Eix posee una titulación presentada como Comunicación y RR.PP., no específica de comunicación publicitaria. Por otra parte, observamos la oferta de dobles grados: Lleida ofrece Comunicación y Periodismo Audiovisuales, al igual que sucede en Sevilla, si bien la denominación es Periodismo y Comunicación Audiovisual; la Rey Juan Carlos hace lo propio aunque de forma más amplia y diversa: Comunicación Audiovisual y Dirección y Administración de Empresas, Periodismo y Comunicación Audiovisual, Publicidad y RR.PP. y Administración y Dirección de Empresas, Publicidad y RR.PP. y Marketing; y la Universidad de Cádiz ofrece el grado doble en Publicidad y RR.PP. y en Turismo y Publicidad y RR.PP. y Marketing e Investigación de Mercado. Por otra parte, tanto la Universidad Internacional de Andalucía, como la Menéndez Pelayo no imparten grados, solo títulos propios de masters y posgrados, así como cursos de especialización o de verano. 
En cuanto a las especificidades que debemos aclarar sobre la oferta de las universidades privadas se observa, en primer lugar, la oferta genérica de un grado en Comunicación hallada IE University o La Rioja. En segundo lugar, advertimos una vasta oferta de títulos propios ${ }^{4}$ relacionados con la comunicación o el diseño y, en tercer lugar, la posibilidad de estudiar grados dobles o triples, a través de una amplia variedad

Tabla 2. Universidades españolas que ofrecen estudios en comunicación

\begin{tabular}{|c|c|c|c|}
\hline Universidad (de titularidad pública) & Publicidad & Periodismo & CAV \\
\hline Alacant & $\mathrm{X}$ & & \\
\hline Alcalá & & & $\mathrm{X}$ \\
\hline Autònoma de Barcelona & $\mathrm{X}$ & $\mathrm{X}$ & $\mathrm{X}$ \\
\hline Barcelona & & & $\bar{X}$ \\
\hline Burgos & & & $\mathrm{X}$ \\
\hline Cádiz & $\mathrm{X}$ & & \\
\hline Carlos III de Madrid & & $\mathrm{X}$ & $\mathrm{X}$ \\
\hline Castilla La Mancha & & $\mathrm{X}$ & \\
\hline Complutense de Madrid & $\mathrm{X}$ & $\mathrm{X}$ & $\mathrm{X}$ \\
\hline Coruña & & & $\mathrm{X}$ \\
\hline Extremadura & & & $\mathrm{X}$ \\
\hline Girona & $\mathrm{X}$ & & $\mathrm{X}$ \\
\hline Granada & & & $\mathrm{X}$ \\
\hline Jaume I de Castellón & $\mathrm{X}$ & $\mathrm{X}$ & $\mathrm{X}$ \\
\hline La Laguna & & $\mathrm{X}$ & \\
\hline Lleida & & $\mathrm{X}$ & $\mathrm{X}$ \\
\hline Málaga & $\mathrm{X}$ & $\mathrm{X}$ & $\mathrm{X}$ \\
\hline Miguel Hernández D’Eix & & & $\mathrm{X}$ \\
\hline Murcia & $\mathrm{X}$ & $\mathrm{X}$ & $\mathrm{X}$ \\
\hline País Vasco Euskal Herriko Unibertsitatea & $\mathrm{X}$ & $\mathrm{X}$ & $\mathrm{X}$ \\
\hline Politècnica de Catalunya & & & $\mathrm{X}$ \\
\hline Politècnica de València & & & $\mathrm{X}$ \\
\hline Pompeu Fabra & $\mathrm{X}$ & $\mathrm{X}$ & $\mathrm{X}$ \\
\hline Rey Juan Carlos & $\mathrm{X}$ & $\mathrm{X}$ & $\mathrm{X}$ \\
\hline Rovira y Virgili & $\mathrm{X}$ & $\mathrm{X}$ & $\mathrm{X}$ \\
\hline Salamanca & & & $\mathrm{X}$ \\
\hline Santiago de Compostela & & $\mathrm{X}$ & $\mathrm{X}$ \\
\hline Sevilla & $\mathrm{X}$ & $\mathrm{X}$ & $\mathrm{X}$ \\
\hline
\end{tabular}




\begin{tabular}{|c|c|c|c|}
\hline Universitat de les Illes Balears & & $\mathrm{X}$ & $\mathrm{X}$ \\
\hline Valladolid & $\mathrm{X}$ & $\mathrm{X}$ & \\
\hline Vigo & $\mathrm{X}$ & & $\mathrm{X}$ \\
\hline Zaragoza & & $\mathrm{X}$ & \\
\hline Universidad (de titularidad privada) & Publicidad & Periodismo & CAV \\
\hline Abat Oliba CEU & $\mathrm{X}$ & $\mathrm{X}$ & \\
\hline Alfonso X el Sabio & $*$ & $*$ & $*$ \\
\hline Antonio de Nebrija & $\mathrm{X}$ & $\mathrm{X}$ & $\mathrm{X}$ \\
\hline Camilo José Cela & $\mathrm{X}$ & $\mathrm{X}$ & $\mathrm{X}$ \\
\hline Cardenal Herrera CEU & $\mathrm{X}$ & $\mathrm{X}$ & $\mathrm{X}$ \\
\hline Católica San Antonio de Murcia & $\mathrm{X}$ & $\mathrm{X}$ & $\mathrm{X}$ \\
\hline Católica de Valencia San Vicente Mártir & & & $*$ \\
\hline Europea de Madrid & * & $\mathrm{X}$ & $\mathrm{X}$ \\
\hline Europea Miguel de Cervantes & $\mathrm{X}$ & $\mathrm{X}$ & $\mathrm{X}$ \\
\hline Francisco de Victoria & $\mathrm{X}$ & $\mathrm{X}$ & $\bar{X}$ \\
\hline IE University & $*$ & $*$ & $*$ \\
\hline Internacional de Catalunya & $\mathrm{X}$ & $\mathrm{X}$ & $\mathrm{X}$ \\
\hline Internacional de La Rioja & $*$ & $*$ & $*$ \\
\hline Mondragon Univertsitatea & & & $\bar{X}$ \\
\hline Navarra & $\mathrm{X}$ & $\mathrm{X}$ & $\mathrm{X}$ \\
\hline Oberta de Catalunya & $*$ & $*$ & $*$ \\
\hline Pontificia de Salamanca & $\mathrm{X}$ & $\mathrm{X}$ & $\mathrm{X}$ \\
\hline Ramón Llul & $\mathrm{X}$ & $\mathrm{X}$ & $*$ \\
\hline San Jorge & $\mathrm{X}$ & $\mathrm{X}$ & $\mathrm{X}$ \\
\hline San Pablo CEU & $\mathrm{X}$ & $\mathrm{X}$ & $\mathrm{X}$ \\
\hline Udima & & $\mathrm{X}$ & \\
\hline Vic & $\mathrm{X}$ & $\mathrm{X}$ & $\mathrm{X}$ \\
\hline
\end{tabular}

Fuente: Elaboración propia a partir de la página web universidad.es (Ministerio de Educación, Cultura y Deporte) y las webs corporativas de cada Universidad

Del cómputo total de universidades, tal y como hemos indicado previamente, el grado en Publicidad es propuesta por un total de 29 universidades, de las cuales 15 son públicas y 14 privadas. La característica clave se encuentra en el hecho de que de entre todas estas titulaciones la materia de Cine y Publicidad únicamente es incluida en el plan de estudios de la Universidad de vigo.

Debemos tener en consideración que el cine como medio consiguió aumentar su inversión publicitaria en los últimos años de acuerdo con el estudio Infoadex ${ }^{5}$. A pesar de las cifras de decrecimiento de los medios convencionales consigue imponerse, junto con internet, como los dos únicos medios de este tipo que lo logran. Así, 
si bien su porcentaje de inversión es bajo en comparación con el resto, su auge como medio publicitario muestra el atractivo de sus posibilidades por parte de las agencias y evidentemente de los anunciantes, atractivo que no resulta acorde, tal y como comprobamos, con la situación en el sistema universitario español

\section{Metodología docente, planning y sistema de evaluación en la oferta de la uvigo}

La aportación que incluimos obedece a la guía docente diseñada para la materia Cine y Publicidad impartida en el primer semestre del cuarto curso del Grado en Publicidad y RR.PP. de la UVigo, como oferta de optatividad, durante el periodo académico 2013/2014. Sus contenidos se estructuran a partir de cuatro grandes bloques de los que surgen 17 temas (cfr. Tabla 3).

Tabla 3. Bloques y temas de contenido de la materia Cine y publicidad

\begin{tabular}{|c|c|}
\hline Bloque de contenido & Temas \\
\hline $\begin{array}{l}\text { 1. Estilos técnico- } \\
\text { narrativos en el ámbito } \\
\text { cinematográfico. }\end{array}$ & $\begin{array}{l}\text { 1. Origen y evolución de la imagen cinematográfica. } \\
\text { 2. Los pioneros: Francia, Italia, Dinamarca, América. } \\
\text { 3. Las escuelas de arte mudo: Escuela Americana, el Expresionismo } \\
\text { alemán, la Escuela Soviética, la Escuela Impresionista y el } \\
\text { Surrealismo. } \\
\text { 4. La creación de Hollywood, las majors y el cine clásico MRP vs } \\
\text { MRI. } \\
\text { 5. El contexto de los años } 40 \text { y el Neorrealismo italiano. } \\
\text { 6. Los nuevos cines: Nouvelle Vague, Free Cinema y el Nuevo Cine } \\
\text { Español. }\end{array}$ \\
\hline $\begin{array}{l}\text { 2. Relaciones entre el } \\
\text { cine y la publicidad } \\
\text { I. Perspectiva desde } \\
\text { la creatividad y la } \\
\text { imitación }\end{array}$ & $\begin{array}{l}\text { 7. La imitación del cine y la incorporación de imágenes } \\
\text { cinematográficas en la publicidad. } \\
\text { 8. El arquetipo del star system cinematográfico en la publicidad. } \\
\text { 9. Incorporación de imágenes cinematográficas y elementos de la } \\
\text { escenificación en la publicidad y viceversa. } \\
\text { 10. Intercambio en las prácticas de dirección. }\end{array}$ \\
\hline $\begin{array}{l}\text { 3. Relaciones entre el } \\
\text { cine y la publicidad II. } \\
\text { La publicidad como } \\
\text { fórmula de financiación }\end{array}$ & $\begin{array}{l}\text { 11. La imagen cinematográfica como soporte publicitario: evolución. } \\
\text { 12. La inserción de publicidad en el cine: la práctica del } \\
\text { emplazamiento. } \\
\text { 13. La técnica del brand entertainment y sus posibilidades. }\end{array}$ \\
\hline $\begin{array}{l}\text { 4. Relaciones entre el } \\
\text { cine y la publicidad } \\
\text { III. Estrategias de } \\
\text { promoción publicitaria } \\
\text { del cine }\end{array}$ & $\begin{array}{l}\text { 14. Planificación de campañas publicitarias. } \\
\text { 15. Herramientas y actividades promocionales específicas en cine. } \\
\text { 16. La incorporación de nuevos medios y nuevas fórmulas } \\
\text { promocionales. } \\
\text { 17. Los eventos cinematográficos y las entregas de premios: } \\
\text { protocolo ceremonial, relaciones públicas y moda. }\end{array}$ \\
\hline
\end{tabular}

Fuente: Guía docente de Cine y Publicidad del Grado en Publicidad y RR.PP. de la Universidad de 


\subsection{Descripción de la metodología docente utilizada}

La materia Cine y Publicidad ofrece un variado conjunto de competencias, algunas de ellas de carácter genérico relacionadas con las competencias de la titulación y otras más específicas (Tabla 4). Así, para el desarrollo de estas competencias se ha diseñado una metodología docente que comprende sesiones magistrales, la elaboración de un proyecto de curso, el estudio de caso o análisis de situaciones, debates y presentaciones orales. Las sesiones magistrales se utilizan para elaborar y explicar los contenidos teóricos acompañando estas disertaciones con la proyección de material gráfico y audiovisual a modo de ejemplos con los que poder identificar y comprender los conceptos y prácticas explicitadas durante la sesión. Por otra parte, se solicita el desarrollo de un proyecto cuyo seguimiento será realizado en las horas de seminarios; mientras que durante las sesiones prácticas se elaborarán diversas tareas, como debates o foros de discusión sobre determinados aspectos incluidos en los contenidos de la materia o el estudio de casos a través de comentarios (escritos u orales).

Tabla 4. Relación de competencias específicas desarrolladas con la materia Cine y Publicidad

\begin{tabular}{|c|c|}
\hline Competencia & Tipología \\
\hline $\begin{array}{l}\text { Competencia de los procesos evolutivos a nivel tecnológico, cultural y } \\
\text { narrativo de la imagen audiovisual }\end{array}$ & \multirow[t]{4}{*}{ Saber } \\
\hline Conocimiento de la evolución histórica del cine y de la publicidad. & \\
\hline $\begin{array}{l}\text { Conocimiento técnico-narrativo de la imagen en las diferentes } \\
\text { escuelas cinematográficas e influencia en el cine publicitario }\end{array}$ & \\
\hline $\begin{array}{l}\text { Conocimiento de las diferentes modalidades y técnicas de creación } \\
\text { publicitaria, desde el recurso creativo del cine }\end{array}$ & \\
\hline $\begin{array}{l}\text { Capacidad para analizar y aplicar recursos creativos de la publicidad } \\
\text { en el ámbito cinematográfico }\end{array}$ & \multirow[t]{2}{*}{ Saber hacer } \\
\hline $\begin{array}{l}\text { Capacidad creativa de generar nuevas formas de inserción publicitaria } \\
\text { en la imagen cinematográfica }\end{array}$ & \\
\hline $\begin{array}{l}\text { Capacidad de analizar los elementos de la comunicación y generar los } \\
\text { conocimientos de esta disciplina }\end{array}$ & \multirow[t]{5}{*}{ Saber estar/ser } \\
\hline $\begin{array}{l}\text { Capacidad para administrar el tiempo, con habilidad para la } \\
\text { organización de tareas }\end{array}$ & \\
\hline $\begin{array}{l}\text { Capacidad de adaptarse hacia los nuevos recursos creativos que } \\
\text { generan las nuevas imágenes y los nuevos soportes }\end{array}$ & \\
\hline $\begin{array}{l}\text { Capacidad para trabajar en equipo en el ámbito de la producción del } \\
\text { cine publicitario }\end{array}$ & \\
\hline $\begin{array}{l}\text { Capacidad de adaptación a los objetivos organizativos y de trabajo en } \\
\text { equipo }\end{array}$ & \\
\hline
\end{tabular}

Fuente: Guía docente de Cine y Publicidad del Grado en Publicidad y RR.PP. de la Universidad de 
Por último debemos hacer constar las posibilidades de tutorización de las que dispone el alumno: en primer lugar, las horas de tutorías presenciales en el despacho del responsable de la materia o en aquellas sesiones de seminarios diseñadas para este fin; y, por ora parte, las opciones online o virtuales, como el correo electrónico o la plataforma de docencia virtual (Faitic) de la propia universidad

\subsection{Sistema de evaluación del alumno y planificación temporal}

Tras la reflexión sobre nuevas metodologías docentes desde la instauración del EEES (VV.AA., 2012) hemos comprendido que en el contexto actual se intenta evitar el simple trasvase de conocimientos de docente a alumno en sesiones magistrales sino que se procura el planteamiento de tareas diversas para el desarrollo de la capacidad crítica y reflexiva del alumno y la autoconstrucción de su propio conocimiento. La finalidad reside, por tanto, en un aprendizaje activo. En todo esto, las TIC' ofrecen distintas posibilidades ${ }^{6}$, configurándose no solo como el acceso a fuentes de información sino que garantizan fórmulas de comunicación más directas y cercanas entre alumno y profesor ayudando a una tutorización más efectiva y constante.

El sistema de evaluación utilizado (cfr. Tabla 5) se ha configurado a través de una evaluación continua en la que entre el proyecto de curso (50\%), las distintas tareas propuestas en las sesiones prácticas $(20 \%)$ y la asistencia y participación (10\%) puede suponer que el alumno obtenga una valoración total de un $80 \%$ (reservando, por tanto, tan solo dos puntos para valorar el examen final). Se ha optado por el empleo del ABP como metodología docente precisamente por sus ventajas, ya que promueve un aprendizaje activo, facilita el desarrollo de competencias transversales como el trabajo en grupo, así como el aprendizaje autónomo o la comunicación y motiva a los estudiantes (Valero García, 2009: 10) a través del conocimiento de casos realistas y destacados en un determinado ámbito. Por ello se plantea el desarrollo de un proyecto de investigación de tipo académico o de aplicación práctica lo que les ayudará en la posterior elaboración del Trabajo de Fin de Grado durante el segundo semestre del curso. El proyecto versará sobre algún contenido específico de la materia - con la condición obligatoria de que aborde alguna de las interrelaciones entre cine y publicidad-, que se concreta entre profesor y alumno, y se materializa en una entrega escrita (si bien se podría optar por tres entregas parciales, siendo la primera el planteamiento del objeto de estudio y desarrollo del marco teórico, la segunda la relación de hipótesis de partida y el diseño metodológico y una última con el proyecto completo) y en una presentación oral.

Tabla 5. Métodos de evaluación del alumno

\begin{tabular}{|c|c|c|}
\hline Método & Descripción & Valoración \\
\hline Asistencia y participación & $\begin{array}{l}\text { Valoración de la asistencia y la participación } \\
\text { del alumno }\end{array}$ & $10 \%$ \\
\hline Proyecto & $\begin{array}{l}\text { Trabajo de investigación o de carácter teórico- } \\
\text { práctico }\end{array}$ & $45 \%$ \\
\hline
\end{tabular}




\begin{tabular}{|l|l|l|}
\hline Estudio de casos & $\begin{array}{l}\text { Elaboración de análisis sobre distintos casos o } \\
\text { problemáticas }\end{array}$ & $15 \%$ \\
\hline Debates & $\begin{array}{l}\text { Discusión mediante división previa en grupos } \\
\text { (a favor o en contra) o foro de discusión } \\
\text { grupal sobre alguna de las posibilidades de } \\
\text { interacción entre la disciplina publicitaria y la } \\
\text { cinematográfica }\end{array}$ & $5 \%$ \\
\hline Pruebas & $\begin{array}{l}\text { Examen escrito final de los contenidos teóricos } \\
\text { de la materia }\end{array}$ & $20 \%$ \\
\hline Presentaciones & $\begin{array}{l}\text { Exposición oral del proyecto o de otras tareas } \\
\text { designadas }\end{array}$ & $5 \%$ \\
\hline
\end{tabular}

Fuente: Elaboración propia

A este proyecto se unen una serie de estudios de caso que serán desarrollados en las sesiones prácticas. Detallamos (Tabla 6) la relación de tareas en su integración en el cronograma de la materia7

Tabla 6. Cronograma de tareas

\begin{tabular}{|l|l|l|}
\hline \multicolumn{1}{|l|}{ Sem. } & Tipo & $\begin{array}{l}\text { Contenido } \\
\text { Conformación de grupos de trabajo y delimitación inicial del tema del } \\
\text { proyecto }\end{array}$ \\
\hline 4 & Seminario 1 & $\begin{array}{l}\text { Estudio de caso 1. "Cousas do Kulechov": el cine soviético y la } \\
\text { importancia del montaje }\end{array}$ \\
\hline 5 & Práctica 2 & $\begin{array}{l}\text { Estudio de caso 2. Presentación de una de las películas representativas } \\
\text { de los Nuevos Cines }\end{array}$ \\
\hline 6 & Seminario 2 & Sesión de seguimiento del proyecto \\
\hline 7 & Práctica 3 & $\begin{array}{l}\text { Estudio de caso 3 (sesión 1). Influencias cinematográficas en piezas } \\
\text { publicitarias }\end{array}$ \\
\hline 8 & Práctica 4 & Estudio de caso 3 (sesión 2). \\
\hline 9 & Práctica 5 & Estudio de caso 4 (sesión 1). Campaña publicitaria de un film \\
\hline 10 & Seminario 3 & Sesión de seguimiento del proyecto \\
\hline 11 & Práctica 6 & Entrega 1 proyecto. Objeto de estudio y marco teórico \\
\hline 12 & Seminario 4 & Estudio de caso 4 (sesión 2). \\
\hline 13 & Práctica 7 & Sesión de seguimiento del proyecto \\
\hline 14 & Práctica 8 & Entrega 2 proyecto. Hipótesis de partida y diseño metodológico. \\
\hline 15 & Seminario 5 & $\begin{array}{l}\text { Debate sobre cine y publicidad. Fórmulas publicitarias para la } \\
\text { promoción de un film. Medios convencionales VS medios no } \\
\text { convencionales }\end{array}$ \\
\hline & & Entrega final del proyecto \\
\hline
\end{tabular}




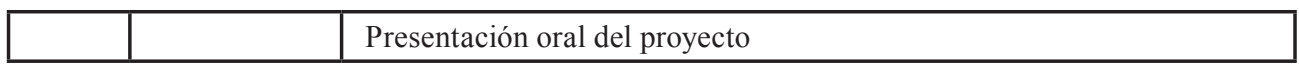

Fuente: Elaboración propia

\section{Conclusiones}

A través de la investigación realizada y presentada en el texto de este artículo se muestra la ausencia de la materia Cine y Publicidad — así como de contenidos específicos sobre la interrelación entre estos dos campos de conocimiento- en los planes de estudio de los grados en Publicidad y RR.PP. de las universidades españolas, sin distinción en función de su titularidad (pública o privada), a pesar del actual crecimiento observado en las cifras de inversión publicitaria en el medio cinematográfico (lo que confiere relevancia a su estudio en profundidad). Esta materia únicamente se hallaría en el grado implantado en la Universidade de Vigo como una oferta de optatividad para el alumno. Con este apunte inicial, podríamos señalar como primera conclusión el hecho de que las universidades caminarían varios pasos por detrás de la actualidad en el ámbito de la comunicación.

Lo cierto es que las universidades han focalizado su atención en los últimos años en las opciones que se consiguen con nuevas herramientas publicitarias y comunicativas, especialmente aquellas que han nacido a través de internet, entre las que sobresalen las social networks. Esto ha sido consecuencia no sólo a raíz del incremento en cuanto a número de usuarios, sino por la creación de nuevos perfiles laborales como el de community manager, actualmente en auge y en torno al que ha surgido una extensa oferta formativa. No obstante, consideramos que la materia Cine y Publicidad incluye contenidos válidos y relevantes por lo que su inclusión en los planes de estudio resulta positiva. No debemos, como docentes, pecar y olvidarnos del estudio en profundidad de cada uno de los medios convencionales, entre ellos el cine, para afrontar las opciones y ventajas que ofrecen desde un punto de vista publicitario. Debemos formar comunicadores polivalentes.

En otro orden de cuestiones, no hemos podido realizar finalmente una comparativa entre guías docentes de la materia objeto de estudio precisamente por su ausencia en los planes de estudio de las titulaciones en Publicidad en España. Se advierte, sin embargo, la oferta de otra tipología de materias sobre el conocimiento y funcionamiento del ámbito audiovisual, como es el caso de Tecnología de los Medios Audiovisuales o Teoría y Práctica de la Comunicación Televisiva, etc. desde un punto de vista esencialmente práctico; así como otras materias de carácter más teórico como es el caso de Narrativa Audiovisual (si bien con escasa presencia). A pesar de ello, con estas posibilidades no se podrían conocer las características que posee el cine y que han influido en la publicidad así como las distintas relaciones o influencias que se ejercen entre una y otra disciplina, tal y como hemos puntualizado en el epígrafe de explicación de diseño de la guía docente y en concreto en la definición de contenidos.

Podemos concluir, por tanto, que la Universidad de Vigo se configura como pionera en este sentido, atendiendo a las opciones de este medio convencional que 
había estado expuesto a una situación de cierto abandono y desinterés por parte de las propias agencias publicitarias y por parte de los anunciantes que no entendían o no querían ver la eficacia y los resultados que pueden obtenerse a través de la interacción con este medio.

\section{Referencias bibliográficas}

ANECA (2008). Libro Blanco. Títulos de Grado en Comunicación. ANECA. Madrid. Versión online disponible en: http://www.aneca.es/activin/activin_conver_LLBB. asp.

PÉREZ, M. T.; ARRATIA, O.; MARTÍN, M. A.; GALISTEO, D. (2009). Innovación en docencia universitaria con Moodle. Casos prácticos. Alicante: Editorial Club Universitario.

TORRES ROMAY, E. (2006). "Formación universitaria en estrategias publicitarias. Problemas y perspectivas. Algunas aportaciones desde el alumnado". En CARCELÉN GARCÍA, S.; RODRÍGUEZ WAMGÜERMERT, C.; VILLAGRA, N.. Propuestas para una comunicación de calidad. Contenidos y efectos de la formación. Madrid: Edipo. pp. 613-620.

VALERO-GARCÍA, M. (2009). A aprendizaxe baseada en proxectos no ensino universitario. Vigo: Universidade de Vigo, Vicerreitoría de Formación e Innovación Educativa.

VV.AA. (2012). Innovar na universidade: experiencias do profesorado. Vigo: Universidade de Vigo, Vicerreitoría de Formación e Innovación Educativa

\section{Notas}

1. Sobre los antecedentes de los actuales estudios de comunicación, la ANECA en su Libro Blanco sobre Comunicación establece que podemos encontrarlos "en las Escuelas de periodismo, de radio y televisión y de publicidad que funcionaron durante el franquismo y que posteriormente desencadenaron en las actuales facultades de Ciencias de la Información y de la Comunicación. Es cierto que anteriormente a este periodo existen otras iniciativas que ponen las bases de estos estudios como es el caso del curso de periodismo organizado por el catedrático y periodista Fernando Araujo y Gómez, en la Universidad de Salamanca, en el año 1887”. En ANECA (2008). Libro Blanco. Títulos de Grado en Comunicación. ANECA. Madrid. Versión online disponible en: http://www.aneca.es/activin/activin_conver_LLBB.asp [04/08/2013].

2. Universidades públicas españolas que no ofrecen estudios en comunicación: Almería, Autónoma de Madrid, Cantabria, Córdoba, Huelva, Internacional de Andalucía, Internacional Menéndez Pelayo, Jaén, La Rioja, Las Palmas de Gran Canaria, León, Oviedo, Pablo de Olavide, Politécnica de Cartagena, Politécnica de Madrid, Pública de Navarra y la Uned.

3. Universidades privadas españolas que no ofrecen estudios en comunicación: Católica de Ávila, Internacional Valenciana y Pontificia de Comillas.

4. En el caso de la Abat Oliba CEU se ofrece un programa de Especialización en Comunicación Digital así como el título propio Diseño Gráfico y Multimedia; la Católica de Valencia San Vicente Mártir se ofrece Multimedia y Artes Digitales; la Oberta de Catalunya dos titulaciones propias, Comunicación 
y Multimedia; Ramón Llul Cine y Televisión, en lugar de Comunicación Audiovisual; Antonio de Nebrija incluye Cine y Series de Ficción; Camilo José Cela oferta Cine así como Protocolo y Organización de Eventos; o la Universidad Francisco de Victoria que oferta uno de los listados más amplios, Social Media Management, Dirección de Comunicación, Animación y Aplicaciones 3D en Tiempo Real, Realización y Producción de Nuevos Formatos en TV y Cine Digital, Marketing Digital, Diseño Gráfico Multimedia e Interactivo, Diseño de Moda.

5. Cfr. Estudio de la inversión publicitaria en España 2011, 2012 y 2013. En la página web corporativa de Infoadex http://www.infoadex.es.

6. Más ventajas sobre el uso de las TIC' en el ámbito educativo, cfr. PÉREZ, M.T.; ARRATIA, Ó.; MARTÍN, M. A.; GALISTEO, D. (2009).

7. La primera semana de clase se realiza la presentación y la segunda únicamente se lleva a cabo la primera sesión de tipo teórico.

\section{La autora}

Silvia García Mirón, Profesora ayudante del Departamento de Comunicación Audiovisual y Publicidad de la Universidade de Vigo. Licenciada en Publicidad y RRPP (2006) y Comunicación Audiovisual (2009) por la Universidade de Vigo. Entre sus principales líneas de investigación destacan el análisis de estrategias de programación de las emisoras televisas; el estudio de nuevas técnicas de fidelización del telespectador; la planificación y planteamiento de estrategias publicitarias en nuevos medios; y el uso y aplicación de las NTICs en el ámbito docente. Desarrollando su tesis doctoral centrada en un análisis sobre los contenidos y las estrategias de programación en prime time de Antena 3, primera emisora privada de televisión española 